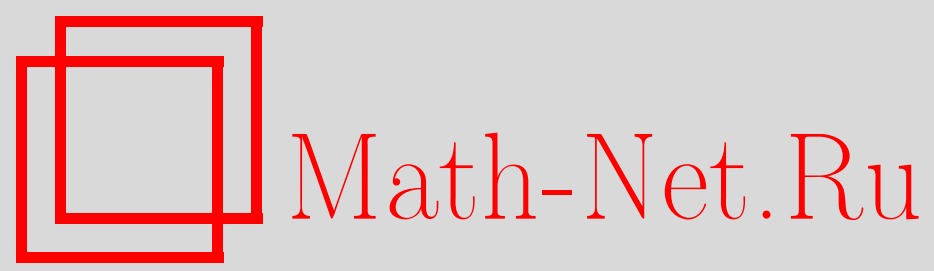

Н. О. Седова, С. В. Токмаков, Об использовании нейроуправления с запаздывающей обратной связью в задаче стабилизации по выходу, Нечеткие системы и мягкие вычисления, 2020, том 15, выпуск 1, 26-42

DOI: https://doi.org/10.26456/fssc66

Использование Общероссийского математического портала Math-Net.Ru подразумевает, что вы прочитали и согласны с пользовательским соглашением

http: //www.mathnet.ru/rus/agreement

Параметры загрузки:

IP : 54.237 .59 .107

26 апреля 2023 г., 04:38:04 
УДК 531.36, 004.032.26

\section{ОБ ИСПОЛЬЗОВАНИИ НЕЙРОУПРАВЛЕНИЯ С ЗАПАЗДЫВАЮЩЕЙ ОБРАТНОЙ СВЯЗЬЮ В ЗАДАЧЕ СТАБИЛИЗАЦИИ ПО ВЫХОДУ}

Седова Н.О., Токмаков С.В.

Ульяновский государственный университет, г. Ульяновск

Поступила в редакиию 30.10.2019, после переработки 29.01.2020.

На примере перевернутого маятника на тележке продемонстрирован метод стабилизации механической системы обратной связью, не зависящей от скоростей. Возможность использования лишь значений координат обеспечивается запаздыванием обратной связи. Конструкция использует нейронную сеть, предполагая известным некоторое стабилизирующее управление по состоянию. Последнее строится в виде управления с переключением, допуская произвольные отклонения маятника от целевого верхнего положения.

Ключевые слова: стабилизация по выходу, механическая система, нейроуправление.

Нечеткие системы и мягкие вычисления. 2020. T. 15, № 1. С. 26-42. https://doi.org/10.26456/fssc66

\section{Введение}

В большинстве задач управления предполагается устойчивость управляемой системы, в том или ином смысле. Зачастую уместным требованием оказывается устойчивость в смысле Ляпунова - свойство системы сохранять заданный режим функционирования при неконтролируемых возмущениях ее состояния. Управление, обеспечивающее системе такое свойство, будем называть стабилизирующим, а задачу синтеза такого управления - задачей стабилизации.

Наиболее эффективной стратегией стабилизации, как правило, обеспечивающей системе дополнительные полезные свойства (например, параметрическую устойчивость) является построение управления по принципу обратной связи. Если управление зависит от всех координат вектора состояния системы, то говорят о стабилизации по состоянию, если же в качестве аргументов обратной связи предполагается использовать лишь часть координат либо какую-то функцию, от этих координат зависящую, то получаем задачу стабилизации по выходу.

Задача стабилизации по состоянию изучена гораздо больше и обладает более широкими возможностями с точки зрения синтеза управления, нежели задача стабилизации по выходу. Однако на практике возникает вопрос реализуемости построенного управления. В реальности измерение далеко не всех параметров системы возможно осуществить. Наличие датчиков в контуре управления может внести дополнительные погрешности в систему, поскольку каждый измеритель представляет собой отдельный динамический объект; пренебрежение соответствующей динамикой может сказаться на адекватности используемой математической модели, а учет переходных процессов в измерителях увеличивает размерность системы, повышая сложность задачи. 
Проблема уменьшения размерности вектора, используемого для построения стабилизирующей обратной связи, исследовалась с помощью различных подходов. Важным частным случаем стабилизируемых систем являются механические системы, для которых возникают сложности с оцениванием текущих значений параметров, в частности, с оцениванием скоростей. Задача стабилизации механической системы без измерения скоростей активно изучается в последние десятилетия; обзор некоторых известных результатов представлен в работе [1].

Один из подходов к решению этой задачи - стабилизация обратной связью с запаздыванием. Использование лишь текущих значений самих функций в структуре обратной связи не позволяет в общем случае стабилизировать систему, описываемую уравнениями второго порядка. Это видно уже на примере задачи стабилизации для простейшего уравнения второго порядка $\ddot{x}(t)=u$. Пропорциональный регулятор $u=k x(t)$, очевидно, не обеспечивает асимптотическую устойчивость нулевого решения ни при каких значениях $k$. Если же управление искать в виде $u=k_{1} x\left(t-\tau_{1}\right)+k_{2} x\left(t-\tau_{2}\right)$ с некоторыми $\tau_{1} \geq 0, \tau_{2}>0$, то параметры характеристического многочлена $h(s)=s^{2}-k_{1} e^{-\tau_{1} s}-k_{2} e^{-\tau_{2} s}$ можно подобрать так, чтобы характеристическое уравнение $h(s)=0$ не имело корней в правой комплексной полуплоскости.

Аналогичный подход можно применить и в задаче стабилизации линейной системы второго порядка произвольной размерности. В статье [2] предложен алгоритм, согласно которому нахождение параметров стабилизирующего управления при заданных величинах запаздываний сводится к решению системы линейных матричных неравенств (ЛМН). При этом считается известным стабилизирующее линейное управление по состоянию, параметры которого участвуют в задании решаемых ЛМН. Описать всё множество решений системы ЛМН возможно лишь в простейших случаях, зато численные решения могут быть легко получены с использованием готовых программных продуктов, в частности, с помощью стандартных инструментов математической среды MATLAB. При рассмотрении задачи в нелинейной постановке на пути реализации описанного подхода возникают две отдельные задачи: 1) построить стабилизирующее управление по состоянию: 2) используя построенное управление в качестве основы, «образца», получить управление в виде обратной связи от заданной части вектора состояния (в том числе его предшествующих значений, ввиду указанной невозможности в общем случае использовать мгновенную обратную связь).

Первая из этих задач, как известно, единого алгоритма решения не имеет. Мы рассмотрим ее на примере стабилизации классической модели «перевернутый маятник на тележке» в верхнем, неустойчивом, положении равновесия. Эта модель, является, как известно, «испытательным полигоном» для разнообразных методов управления, начиная от классического ПИД-регулятора и заканчивая такими современными подходами, как нечеткое управление [3,4] и нейроуправление [5]. Последний подход основан на применении искусственных нейронных сетей (ИНС), способных «обучаться» при наличии «образца». Именно нейроуправление предлагается применить в данной работе для решения второй из указанных задач. Отличительной чертой построенного на выходе управляющего воздействия (которое является приложенной к тележке скалярной силой), является то, что оно строится в виде обратной связи, зависящей от предыдущих координат системы (но не от скоростей; таким образом, не весь вектор состояния считается доступным для измерения). При этом допускаются произвольные отклонения маятника от желаемого равновесия. Заметим, что в результате достигается стабилизация 
по всем координатам системы, в отличие, например, от [5], где для обратной связи используются только значения угла отклонения маятника и угловой скорости, но при этом не достигается стабилизация равновесия тележки.

В первом разделе статьи описывается стабилизирующее управление для линеаризованной модели маятника в виде запаздывающей обратной связи, не зависящей от скоростей. Во втором разделе строится «раскачивающее» управление по состоянию для нелинейной модели в условиях произвольного отклонения маятника от целевого равновесия. Это управление используется для обучения нейронной сети, на выходе которой получается нейроуправление, не зависящее от скоростей, но использующее не текущие, а предшествующие измеренные значения координат маятника и тележки. Обсуждение последнего управления, а также итогового регулятора с переключением между «раскачивающим» и линейным управлениями, составляют содержание третьего раздела. Заключение содержит обобщение полученных результатов.

\section{1. Стабилизирующее управление по выходу с запаздывающей обратной связью для системы «перевернутый маятник на тележке» (линейная модель)}

\section{1. Стабилизачия линейной системы второго порядка общего вида}

Рассмотрим систему линейных дифференциальных уравнений второго порядка:

$$
\ddot{y}(t)=A_{1} y(t)+A_{2} \dot{y}(t)+B u\left(t-\tau_{1}\right),
$$

где $t \in R^{+}=[0,+\infty)$ - независимая переменная (время), $y(t) \in R^{n}$, точка обозначает производную по времени, $u(t) \in R^{k}(k \leq n)$ - вектор управления, при этом управляющее воздействие строится в виде линейной обратной связи с запаздыванием:

$$
u(t)=K_{1} y(t)+K_{2} y(t-\tau),
$$

$\tau, \tau_{1}>0$ - постоянные, $A_{1}, A_{2} \in R^{n \times n}, B \in R^{n \times k}, K_{1}, K_{2} \in R^{k \times n}$ - матрицы.

При отсутствии управления система (1) имеет нулевое (стационарное) решение. Если это решение асимптотически устойчиво (неустойчиво), то будем называть асимптотически устойчивой (неустойчивой) систему (1). Предположим, что при $u(t) \equiv 0$ система $(1)$ неустойчива, и рассмотрим задачу стабилизации в следующей форме: найти управление вида (2) такое, что система (1)-(2) асимптотически устойчива.

Введем обозначения: $x(t)=\left(x_{1}^{\prime}(t), x_{2}^{\prime}(t)\right)^{\prime}=\left(y^{\prime}(t), \dot{y}^{\prime}(t)\right)^{\prime}$ (штрих обозначает транспонирование), $\tau_{2}=\tau+\tau_{1}$. Тогда система (1)-(2) примет следующий вид:

$$
\dot{x}(t)=\left(\begin{array}{rr}
0 & I_{n} \\
A_{1} & A_{2}
\end{array}\right)+\sum_{i=1}^{2} K_{i} x_{1}\left(t-\tau_{i}\right) .
$$

Предположим, что пара матриц $\left(\left(\begin{array}{cc}0 & I_{n} \\ A_{1} & A_{2}\end{array}\right),\left(\begin{array}{c}0 \\ B\end{array}\right)\right)$ стабилизируема (то есть существует матрица $K \in R^{k \times 2 n}$ такая, что матрица $\left(\begin{array}{rr}0 & I_{n} \\ A_{1} & A_{2}\end{array}\right)+\left(\begin{array}{c}0 \\ B\end{array}\right) K$ гурвицева). Матрица $K$ может быть найдена различными способами (см., например, [6]). Здесь мы используем технику сведения синтеза управления к решению системы ЛМН, используемую далее для нахождения параметров управления (2). 
Этот способ получил распространение с появлением эффективных вычислительных процедур для нахождения решений ЛМН. Построение подходящих ЛМН основывается, как правило, на тех или иных достаточных условиях устойчивости (или других свойств). В данном случае, если искать функцию Ляпунова в виде квадратичной формы с (симметричной) матрицей $P$, то условия теоремы Ляпунова об асимптотической устойчивости сведутся к системе матричных неравенств $P>0,(A+B K)^{\prime} P+P(A+B K)<0$ (здесь знак «>» означает положительную определенность матрицы). Эта нелинейная относительно неизвестных матриц $P$ и $K$ система стандартной процедурой приводится к линейной: умножим второе неравенство справа и слева на $P^{-1}$ и обозначим $K P^{-1}=M$, получив в итоге линейную относительно $P^{-1}$ и $M$ систему матричных неравенств, вслед за решением которой находим матрицу $K$ :

$$
\left\{\begin{array}{l}
P^{-1} A^{\prime}+M^{\prime} B^{\prime}+A P^{-1}+B M<0, P^{-1}>0 \\
K=M P
\end{array}\right.
$$

Пусть теперь $\tau_{1}>0$ - заданная постоянная, матрицы $\bar{K}_{1}, \bar{K}_{2}$ - такие, что матрица $\left(\begin{array}{cc}0 & I_{n} \\ A_{1} & A_{2}\end{array}\right)+\left(\begin{array}{c}0 \\ B\end{array}\right)\left(\bar{K}_{1} \tau_{2} \bar{K}_{2}\right)$ гурвицева.

Положим $K_{1}=\bar{K}_{1}+\bar{K}_{2}, K_{2}=-\bar{K}_{2}$ и определим матрицу

$$
D_{1}=\left(\begin{array}{rr}
0 & I_{n} \\
A_{1}+B\left(K_{1}+K_{2}\right) & A_{2}-\tau_{2} B K_{2}
\end{array}\right) .
$$

Рассмотрим также следующие матричные неравенства:

$$
\begin{gathered}
\tau_{2}^{2} K_{2}^{\prime} B^{\prime} S_{2} B K_{2}-4 \tau_{2}^{-2} S_{2}<0 \\
\left(\begin{array}{rrr}
\Phi_{1} & \Phi & \Phi \\
\Phi^{\prime} & -R_{1} & 0 \\
\Phi^{\prime} & 0 & -4 R_{2}
\end{array}\right)<0
\end{gathered}
$$

где $\Phi=D_{1} P(0 B)^{\prime}, \Phi_{1}=D_{1}^{\prime} P+P D_{1}+\operatorname{diag}\left\{\tau_{1}^{2} K_{1}^{\prime} R_{1} K_{1}, \tau_{2}^{4} K_{2}^{\prime} R_{2} K_{2}\right\}$.

$\mathrm{B}$ приведенных обозначениях справедливо следующее утверждение:

Теорема 1 (см. [2]). Пусть матрицы $K_{i} \in R^{k \times n}(i=1,2), \tau_{1} \geq 0, \tau_{2}>0$ таковы, ито матрица $D_{1}$, определяемал соотночением (5), гурвицева. Если существуют положительно определенные $S_{2} \in R^{n \times n}, P \in R^{2 n \times 2 n}, R_{1}, R_{2} \in R^{k \times k}$ такие, что неравенства (6) выполняются, то система (3) асимптотически устойчива; при этом неравенства (6) всегда разрешимы при достаточно малых положительных $\tau_{2}, \tau_{1}=O\left(\tau_{2}^{2}\right)$, если $A_{2}=O\left(\tau_{2}\right)$.

\section{2. Стабилизация модели перевернутого маятника на тележке}

Рассмотрим классическую модель, традиционно называемую «перевернутый маятник на тележке» (Рис. 1). Считаем, что тележка смещается в плоскости качания маятника относительно начала координат на величину $x$ под воздействием внешней силы $F=U(t)$. Из уравнений Лагранжа в переменных $x, \theta$ получим:

$$
\begin{array}{r}
M l \ddot{x} \cos \theta+m l^{2} \ddot{\theta}-m g l \sin \theta=0, \\
(m+M) \ddot{x}+m l \ddot{\theta} \cos \theta-m l \dot{\theta}^{2} \sin \theta=U .
\end{array}
$$




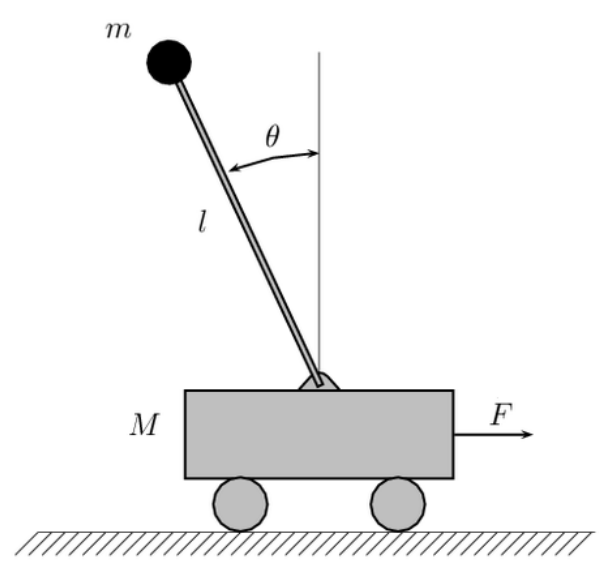

Рис. 1: Перевернутьй маятник на тележке

Здесь $U$ - управляющее воздействие (горизонтальная сила, приложенная к тележке). Выражение (7) можно переписать в виде

$$
\left(\begin{array}{c}
\ddot{\theta} \\
\ddot{x}
\end{array}\right)=\frac{1}{\Delta_{1}}\left(\begin{array}{c}
g(m+M)-m l \dot{\theta}^{2} \cos \theta \\
-m g l \cos \theta+m l^{2} \dot{\theta}^{2}
\end{array}\right) \sin \theta+\frac{1}{\Delta_{1}}\left(\begin{array}{c}
\cos \theta \\
l
\end{array}\right) U
$$

где $\Delta_{1}=l\left(m \sin ^{2} \theta+M\right)$.

Линеаризуем систему (8) в окрестности нулевого равновесия и запишем результат в переменных $y=\left(y_{1}, y_{2}, y_{3}, y_{4}\right)^{\prime}=(x, \theta, \dot{x}, \dot{\theta})^{\prime}$ :

$$
\dot{y}=\left(\begin{array}{cccc}
0 & 0 & 1 & 0 \\
0 & 0 & 0 & 1 \\
0 & -\frac{m g}{M} & 0 & 0 \\
0 & \frac{(m+M) g}{M l} & 0 & 0
\end{array}\right) y+\left(\begin{array}{c}
0 \\
0 \\
\frac{1}{M} \\
-\frac{1}{M l}
\end{array}\right) U(t) .
$$

Используя теорему 1, построим управление для системы (9) в виде линейной обратной связи с запаздыванием:

$$
U(t)=K_{1}\left(x\left(t-\tau_{1}\right) \theta\left(t-\tau_{1}\right)\right)^{\prime}+K_{2}\left(x\left(t-\tau_{2}\right) \theta\left(t-\tau_{2}\right)\right)^{\prime} .
$$

Заметим, что система (9) может быть стабилизирована статической обратной связью по состоянию вида $U(t)=\bar{K}_{1}(x(t) \theta(t))^{\prime}+\tau_{2} \bar{K}_{2}(\dot{x}(t) \dot{\theta}(t))^{\prime}$.

Зафиксируем значение $\tau_{2}=0.01$ и вычислим $\bar{K}_{1}, \bar{K}_{2}$, используя (4) и полагая $\left(\bar{K}_{1} \tau_{2} \bar{K}_{2}\right)=K$. Для численного решения ЛМН применим специальный инструментарий MATLAB, используя значения параметров из [2]: $M=3.9249$, $m=0.2047, l=0.2302, g=9.81$. Затем, применяя теорему 1 , получим допустимый набор остальных параметров управления (10): $\tau_{1}=0.001$, $K_{1}=(15346.24813920 .84), K_{2}=(-15195.789$ - 13276.62) (о значениях запаздываний, при которых ЛМН из теоремы 1 имеют решения для данной задачи, см. [7]).

Выясним теперь, как ведет себя исходная, нелинейная система (8) под действием управления (10). Типичный график численного решения системы (8), 


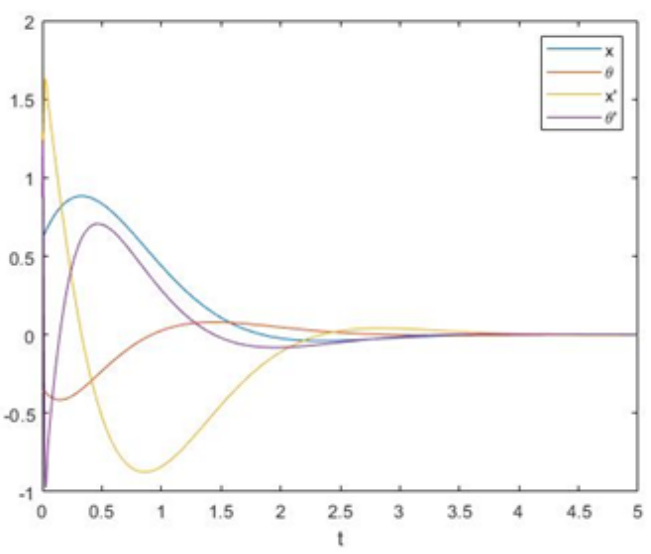

Рис. 2: Графики изменения координат и скоростей системы (8) при управлении (10) c параметрами $\tau_{1}=0.001, \tau_{2}=0.01$, $K_{1}=(15346.24813920 .84), K_{2}=(-15195.789-13276.62)$ и начальными условиями $(x(0), \theta(0), \dot{x}(0), \dot{\theta}(0))=(0.6178,-0.3695,1.2799,0.8734)$

замкнутой управлением (10) с выбранными параметрами $K_{1}, K_{2}, \tau_{1}$ и $\tau_{2}$, построенный с использованием стандартных инструментов MATLAB, представлен на Рис. 2. На основании вычислительных экспериментов установлено, что при заданных значениях параметров линейное управление вида (10) стабилизирует нелинейную систему (8), и область притяжения управляемой системы содержит, по крайней мере, область $Q=\left\{(x, \theta, \dot{x}, \dot{\theta}): \sqrt{x^{2}+\theta^{2}+\dot{x}^{2}+\dot{\theta}^{2}}<2,|\theta|<1.1\right\}$. На Рис. 3 изображены графики решений системы (8), (10), начинающихся вблизи границы области $Q$.
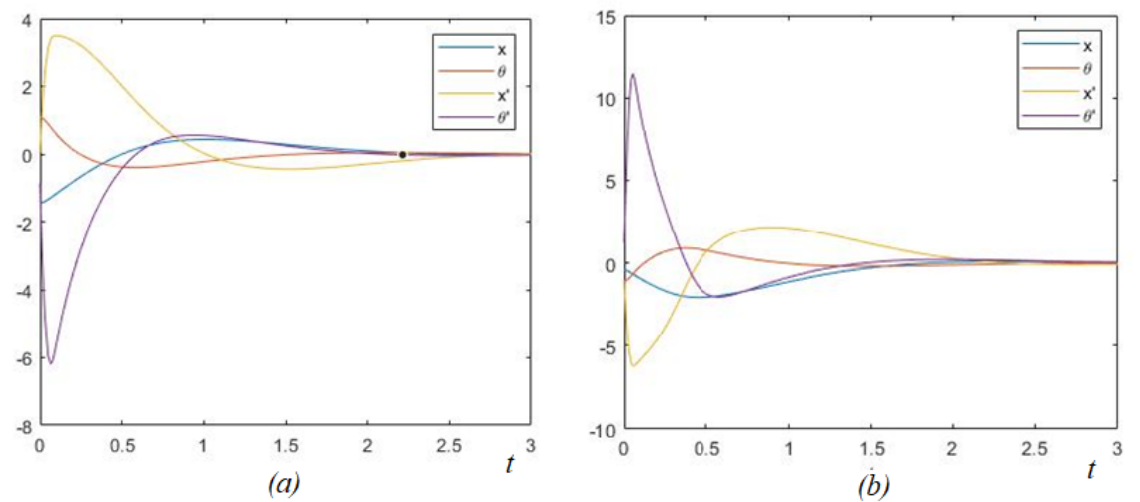

Рис. 3: Графики изменения координат и скоростей системы (8) при управлении (10) с параметрами $\tau_{1}=0.001, \tau_{2}=0.01, K_{1}=(15346.248$ 13920.84), $K_{2}=(-15195.789-13276.62)$ и начальными условиями: (а) $(-1.4391,1.0902,0.0308,-0.8588),(b)(-0.4269,-1.0902,-1.0662,1.2212)$ 


\section{2. Синтез «раскачивающего» управления по состоянию для системы «перевернутый маятник на тележке» (нелинейная модель)}

При использовании линеаризованной системы для решения задачи стабилизации построенное управление в общем случае является стабилизирующим лишь при достаточно малых отклонениях состояния от желаемого равновесия. К примеру, линейным управлением (10) нелинейную систему (8) можно стабилизировать при достаточно малых отклонениях угла (не больших $63^{\circ}$ при рассмотренных значениях параметров). Предполагая, что отклонения от равновесия могут быть произвольными, рассмотрим задачу синтеза стабилизирующего управления с использованием исходной нелинейной модели. Идея состоит в том, чтобы из начального состояния сначала раскачивать маятник, пока текущее состояние не попадет в достаточно малую окрестность желаемого равновесия. После этого можно будет переключиться на линейное управление (10), построенное в предыдущем разделе. Идея такого поэтапного достижения поставленной цели плодотворно используется при управлении нелинейными системами вообще и маятниками в частности; см, например, статью [4], откуда заимствована наглядная иллюстрация данного подхода (Рис. 4).

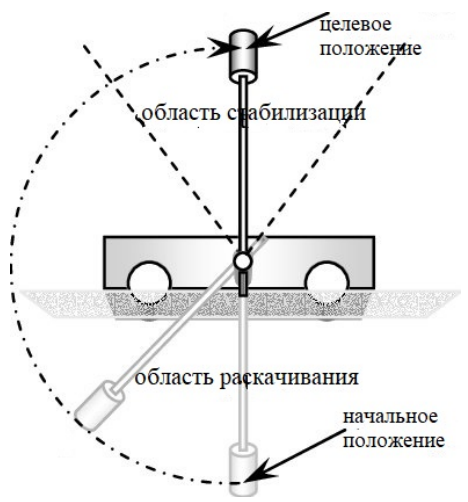

Рис. 4: Раскачивание и стабилизация маятника (зависимость от угла отклонения)

Для построения нелинейного управления с указанными свойствами используем конструкцию из [8]. Прежде всего запишем выражение для полной механической энергии системы (8) (которая в данных предположениях постоянна при отсутствии управления):

$$
E=\frac{(M+m) \dot{x}^{2}}{2}+m l \dot{x} \dot{\theta} \cos \theta+\frac{m l^{2}}{2} \dot{\theta}^{2}+m g l(\cos \theta-1) .
$$

В верхнем положении маятника при условии неподвижности тележки кинетическая энергия равна $T=\frac{m l^{2}}{2} \dot{\theta}^{2}$, так что равенство $E=0$ примет вид:

$$
\frac{m l^{2}}{2} \dot{\theta}^{2}=m g l(1-\cos \theta)
$$

Уравнение (12) описывает фазовую траекторию маятника, соответствующую периодическому движению, проходящему через точку $x=\theta=0$, при этом $\dot{x}=0$ и 
$E=0$. Поэтому для достижения траектории (12) необходимо выполнение условий $\dot{x}=0, x=0, E=0$. Выберем функцию Ляпунова следующего вида:

$$
V=\frac{k_{E}}{2} E^{2}+\frac{k_{v}}{2} \dot{x}^{2}+\frac{k_{x}}{2} x^{2},
$$

где $k_{E}, k_{v}$ и $k_{x}$ - положительные постоянные. Вычисляя производную функции (13), используем (11) и учтем (8). В итоге получим $\dot{E}=U \dot{x}$ и

$$
\dot{V}=\dot{x}\left(k_{E} E U+\frac{k_{v}}{M+m \sin ^{2} \theta}\left(m \sin \theta\left(l \dot{\theta}^{2}-g \cos \theta\right)+U\right)+k_{x} x\right) .
$$

Выберем теперь закон управления таким образом, чтобы производная функции Ляпунова равнялась

$$
\dot{V}=-k_{\sigma} \dot{x}^{2} .
$$

Из (14) и (15) получаем

$$
U=\frac{k_{v} m \sin \theta\left(g \cos \theta-l \dot{\theta}^{2}\right)-\left(M+m \sin ^{2} \theta\right)\left(k_{\sigma} \dot{x}+k_{x} x\right)}{k_{v}+\left(M+m \sin ^{2} \theta\right) k_{E} E} .
$$

Поскольку $\dot{V}$ не является знакоопределенной, для установления асимптотической устойчивости применим теорему Барбашина-Красовского. Заметим, что множество $N=\{(x, \dot{x}, E): \dot{V}=0\}$ может содержать только те решения системы (7), для которых $x \equiv \bar{x}=$ const,$E \equiv \bar{E}=$ const. Анализируя систему (7), нетрудно видеть, что множество $N$ содержит только два ее решения, соответствующих верхнему $(\theta=0)$ и нижнему $(\theta=\pi)$ положениям равновесия маятника.

Заметим также, что если $(x, \theta, \dot{x}, \dot{\theta})=(0, \pi+2 \pi k, 0,0)$, то управление (16) обращается в ноль. Поэтому для выведения системы из этого (устойчивого) положения равновесия необходима дополнительное воздействие $\bar{U} \neq 0$. Выберем для этой цели постоянное воздействие. В численных расчетах возьмем $\bar{U}=5$, которое действует до тех пор, пока выполняются условия $|x|<0.01,|\theta|<0.01,|\dot{\theta}|<0.01$, $|\theta \bmod 2 \pi-\pi|<0.01 ;$ после этого включается управление (16). Заметим, что время, затрачиваемое на «выталкивание» маятника из указанной области, оказывается малым по сравнению с общим временем стабилизации (Рис. 5).

Обсудим параметры управления, которые до сих пор предполагались лишь положительными. Исходя из приведенных рассуждений, единственным дополнительным ограничением оказывается $k_{v}+(M+m \sin \theta) k_{E} E \neq 0$. Поскольку минимальное значение полной энергии равно $-2 M g l$, требуемое неравенство гарантировано при условии $\frac{k_{v}}{k_{E}}>2 m g l(M+m)$. Изменение значений параметров без нарушения данного неравенства приводит к различной продолжительности переходного процесса до достижения цели управления (условием окончания действия управления (16) при выбранных значениях параметров маятника является попадание решения в область $Q$ ). На остальные характеристики переходного процесса указанные изменения не оказывают существенного влияния (см. Рис. 6, 7 , графики решений строились до момента достижения цели управления). Дальнейшие вычисления проводятся при $k_{x}=0.1, k_{v}=4, k_{E}=1, k_{\sigma}=2$. 

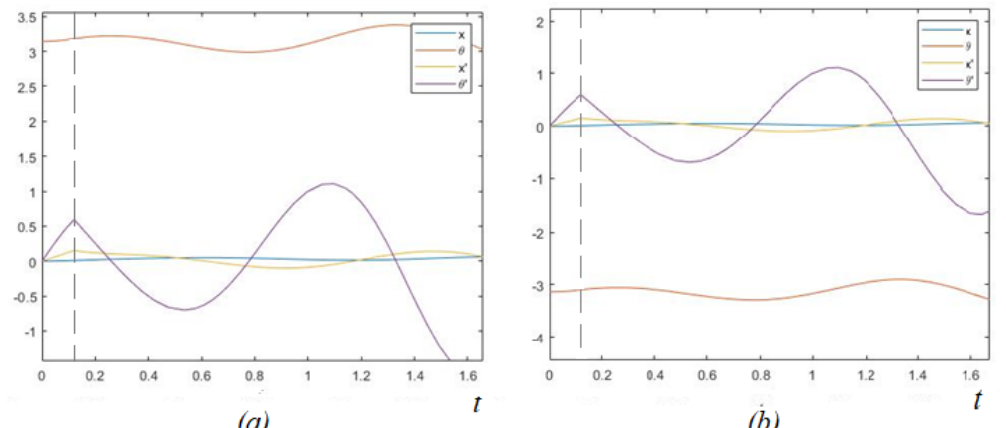

(a)

(b)

Рис. 5: Графики изменения координат и скоростей системы (8) с управлением $U=\bar{U}=5$ при $t<0.1202$ и управлением (16) при $t \geq 0.1202$ с параметрами $k_{x}=0.1, k_{v}=4, k_{E}=1, k_{\sigma}=2$ с начальными условиями: $(a)(0, \pi, 0,0),(b)$ $(0,-\pi, 0,0)$
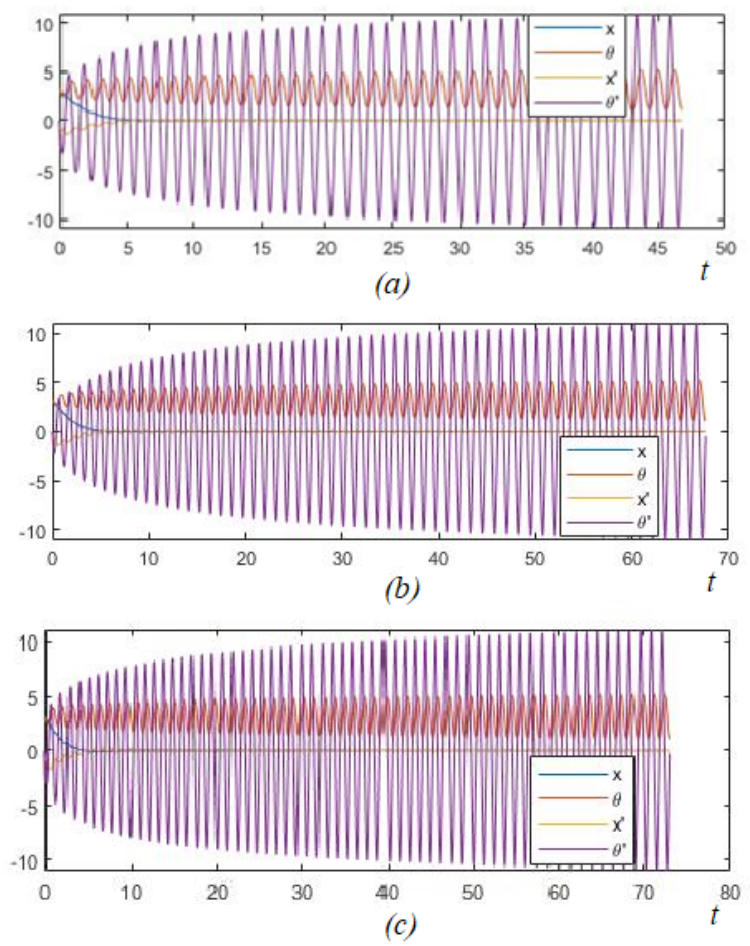

Рис. 6: Графики изменения координат и скоростей системы (8) при управлении (16) с начальным условием $(3, \pi, 0,0)$ и параметрами: $(a) k_{x}=0.1, k_{v}=3.8178$, $k_{E}=1, k_{\sigma}=2.2$; (b) $k_{x}=0.12, k_{v}=4.2, k_{E}=1, k_{\sigma}=2 ;$ (c) $k_{x}=0.1, k_{v}=6.3$, $k_{E}=1.65, k_{\sigma}=2$ 

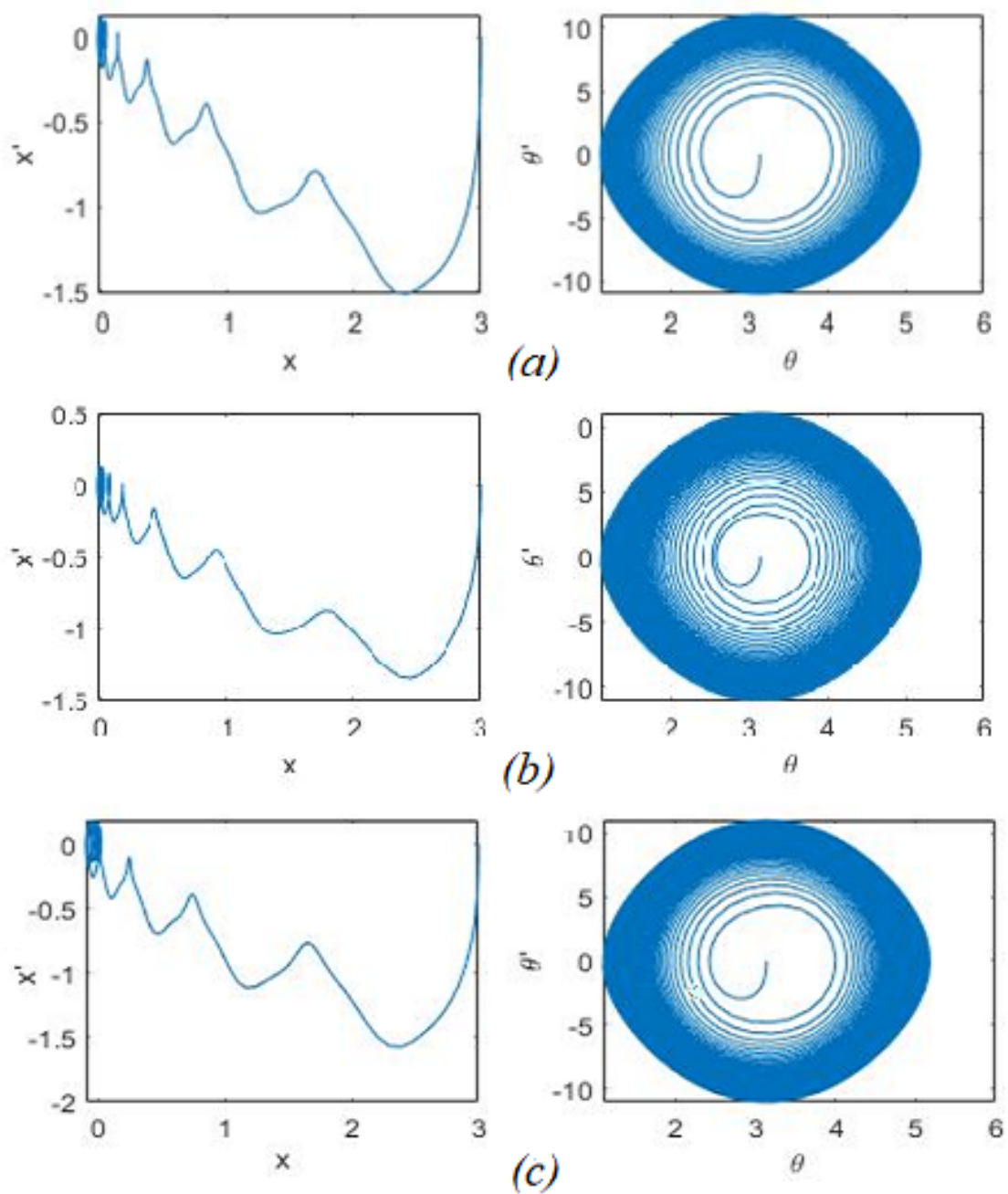

Рис. 7: Плоские сечения Фазового пространства системы (8) при управлении (16), параметры такие же, как на Рис. 6 
3. Стабилизирующее нейроуправление по выходу с запаздывающей обратной связью для системы «перевернутый маятник на тележке» (нелинейная модель)

\section{1. Синтез «раскачивающего» нейроуправления}

На основе построенного в разделе 2 нелинейного управления построим теперь нейроуправление для системы (7) в виде обратной связи, на входе которой выходные данные объекта управления, а на выходе - значения управляющего воздействия $U(t)$. В качестве выходного вектора системы, определяющего обратную связь, используется вектор значений координат в два предшествующих момента времени: $\left(x\left(t-\tau_{1}\right) \theta\left(t-\tau_{1}\right) x\left(t-\tau_{2}\right) \theta\left(t-\tau_{2}\right)\right)^{\prime}$. Обучающая выборка генерируется в результате численного решения уравнений (8) с с управлением по состоянию (16). Схема обучения нейронной сети представлена на Рис. 8.

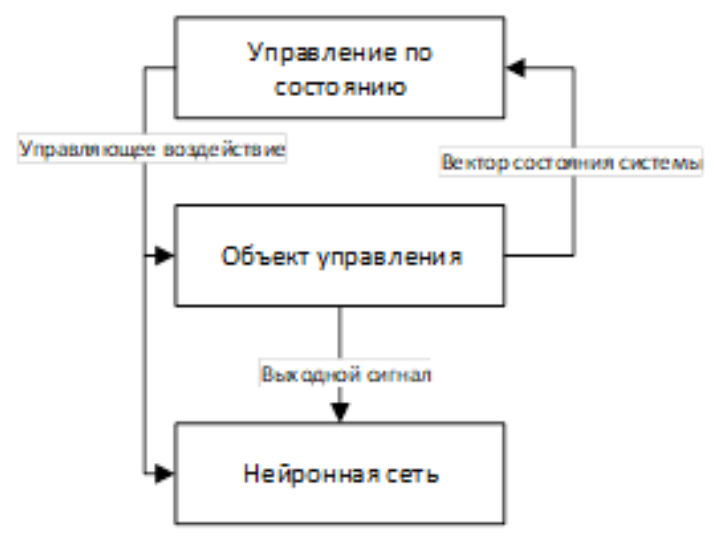

Рис. 8: Схема обучения нейронной сети

Для построения нейроуправления применим нейронную сеть прямого распространения с двумя слоями, для моделирования которой используем стандартные средства пакета Neural Network toolbox системы MATLAB (Рис. 9).

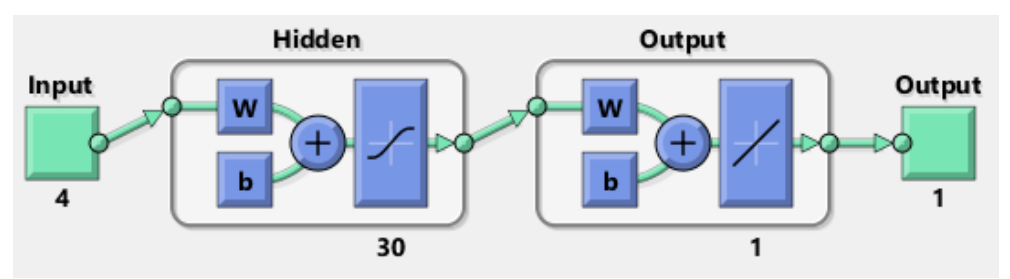

Pис. 9: Архитектура нейронной сети

Расчеты проведем с использованием ранее заданных значений параметров при $\tau_{1}=0.001, \tau_{2}=0.01$. Вычислительные эксперименты показывают эффективность построенного регулятора; пример графика соответствующего численного решения системы (7) до момента попадания в область $Q$ представлен на Рис. 10. 


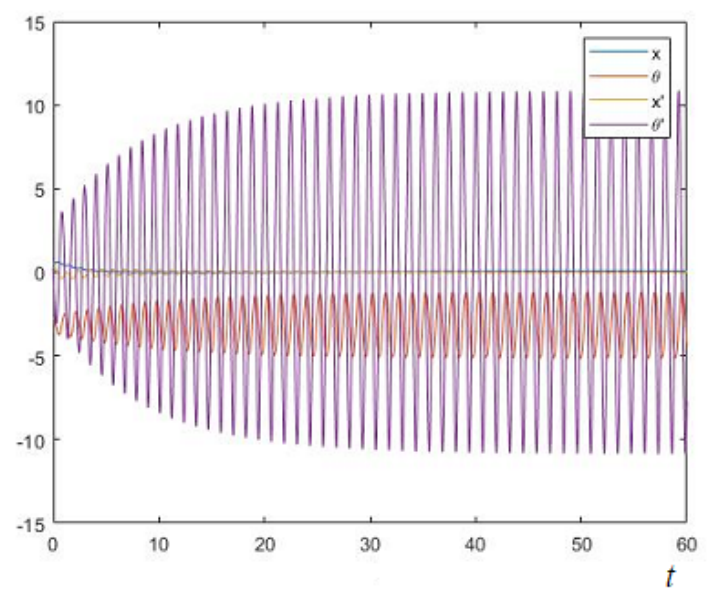

Рис. 10: Решения системы (7) с использованием нейроуправления и начальным условием $(0.5345,-2.792,0.2583,0.2941)$

\section{2. Стабилизирующее управление с переключением}

Окончательное решение задачи приведения маятника из произвольного начального положения в верхнее положение равновесия реализуется путем переключения между построенными управлениями. Линейное управление вида (10) пригодно для стабилизации нелинейной системы лишь в некоторой окрестности нуля, размеры которой зависят от значений параметров и устанавливаются эмпирически (для рассматриваемых значений параметров это область $Q$ ). При больших начальных отклонениях сначала решается задача «раскачивания» маятника нейроуправлением. Как только отклонения переменных состояния системы (7) войдут в область $Q$, включается линейное управление. Если начальное состояние маятника близко к нижнему положению равновесия, необходимо сначала вывести систему из данного равновесия действием дополнительной силы (здесь она принята постоянной).

В результате итоговое управление с переключением окажется обратной связью, определяемой в момент $t$ значениями $\left(x\left(t-\tau_{1}\right) \theta\left(t-\tau_{1}\right) x\left(t-\tau_{2}\right) \theta\left(t-\tau_{2}\right)\right)$. Построенное в разделе 2 нелинейное управление по состоянию используем для обучения нейронной сети, а также для сравнения с итоговым регулятором.

Заметим, что условие переключения управления содержит значения не только координат, но и скоростей, в то время как при управлении по выходу измеряются только координаты системы. Для оценки значений $\dot{x}$ и $\dot{\theta}$ также используем нейросеть. Вычислительные эксперименты показывают эффективность обоих регуляторов и схожесть соответствующих переходных процессов. При использовании нормализации скорость сходимости с нейроуправлением оказывается даже выше по сравнению с управлением по состоянию. На Рис. 11 представлены графики численных решений системы (7) для случайных начальных условий с использованием двух видов управления с переключением: на левых графиках в качестве «раскачивающего» управления используется нейроуправление, на правых - нелинейное управление по состоянию. 

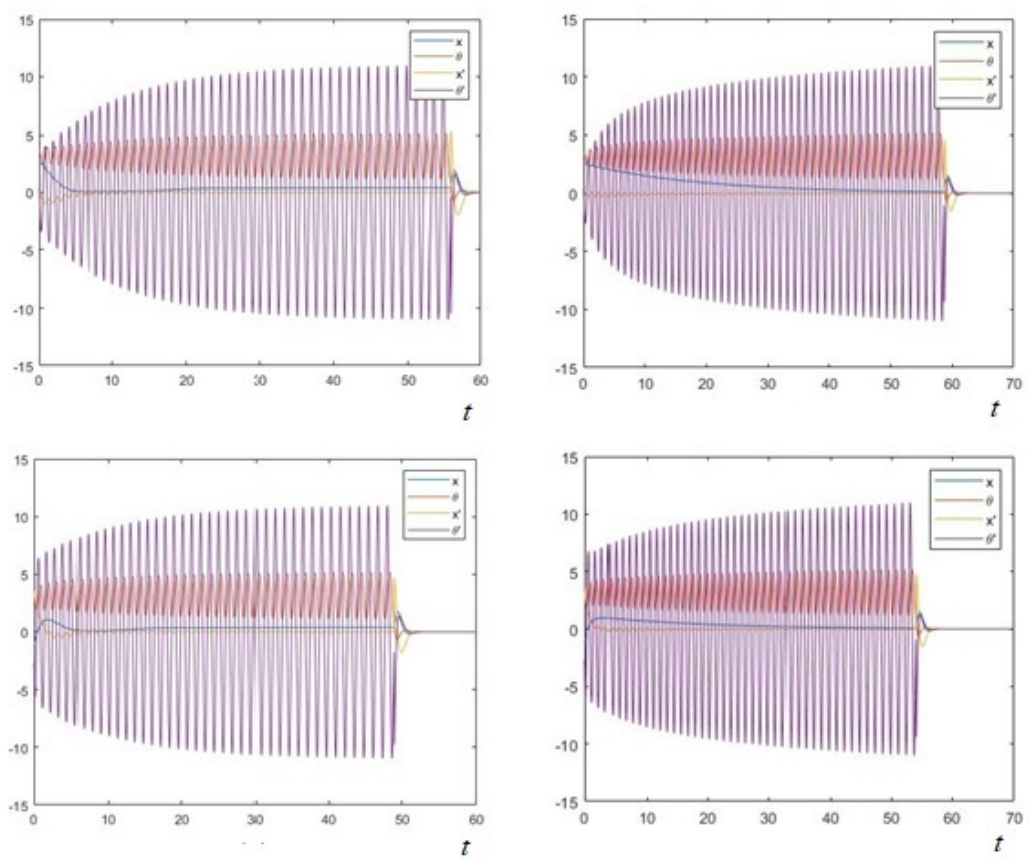

Рис. 11: Решения системы (7) с использованием нейроуправления с переключением по запаздывающему выходу (слева) и нелинейного управления по состоянию (справа)

\section{Заключение}

В работе предлагается управление, стабилизирующее модель «перевернутый маятник на тележке» в верхнем неустойчивом положении равновесия. Особенности построенного управления состоят в следующем:

1. допускаются произвольные начальные отклонения маятника от целевого равновесия;

2. управление строится по выходу, учитываются только два предыдущих положения системы без скоростей;

3. управление предполагает переключение по зависящему от состояния системы условию: сначала маятник раскачивается от начального положения до попадания в окрестность желаемого равновесия, затем включается линейное управление;

4. «раскачивающее» управление строится с использованием нейронной сети, для обучения которой используется заданное нелинейное управление по состоянию;

5. реализация «раскачивающего» управления предполагает возможность совершения маятником полного оборота; 
6. параметры линейного управления находятся из системы ЛМН, допускающих численное решение стандартными алгоритмами (реализованными, в частности, в системе MATLAB).

Сравнение построенного управления со стабилизирующим управлением по состоянию показало эффективность предложенного подхода. Анализ проводился на основе вычислительных экспериментов в системе MATLAB.

Отметим, что и номера измеряемых компонент вектора состояния, и значения запаздываний могут быть заданы нейронной сети в качестве входных параметров (хотя не для любых значений возможно построение стабилизирующего управления, см. [7]).

Полученные результаты демонстрируют одну из возможностей построения стабилизирующего управления для механической системы в виде обратной связи, зависящей только от координат системы и не зависящей от скоростей, с использованием численных методов. Приведенный алгоритм сводится к стандартным вычислительным процедурам, за исключением нахождения нелинейного управления по состоянию, где приходится привлекать эвристические методы (в данной работе для этого используется прямой метод Ляпунова).

\section{Список литературы}

[1] Андреев А.С., Перегудова О.А. Синтез управления двухзвенным манипулятором без измерения скоростей // Автоматизация процессов управления. 2015. T. 42 , № 4. C. $81-89$.

[2] Fridman E., Shaikhet L.E. Delay-induced stability of vector second-order systems via simple Lyapunov functionals // Automatica. 2016. Vol. 74. Pp. 288-296.

[3] Roose A.I., Yahya S., Al-Rizzo H. Fuzzy-logic control of an inverted pendulum on a cart // Computers and Electrical Engineering. 2017. Vol. 61. Pp. 31-47.

[4] Dotoli M., Lino P., Turchiano B. A decoupled fuzzy sliding mode approach to swing-up and stabilize an inverted pendulum // IFAC Proceedings Volumes. 2003. Vol. 36, № 18. Pp. 113-118.

[5] Mladenov V. Application of neural networks for control of inverted pendulum // WSEAS Transactions on circuits and systems. 2011. Vol. 10, № 2. Pp. 49-58.

[6] Зубов В.И. Лекции по теории управления. М.: Наука, 1975.

[7] Токмаков С.В. О решении задачи стабилизации линейной системы запаздывающей обратной связью // Ученые записки УлГУ. Серия: Математика и информационные технологии. 2018. № 1. С. 98-108.

[8] Ченжу Ч. Нелинейное управление перевернутым маятником: ВКР магистра. С.-Пб.: Санкт-Петербургский государственный электротехнический университет «ЛЭТИ»им. В.И.Ульянова (Ленина). 2016.

\section{Образец цитирования}

Седова Н.О., Токмаков С.В. Об использовании нейроуправления с запаздывающей обратной связью в задаче стабилизации по выходу // Нечеткие системы и мягкие вычисления. 2020. Т. 15, № 1. С. 26-42. https://doi.org/10.26456/fssc66 


\section{Сведения об авторах}

1. Седова Наталья Олеговна

профессор кафедры информационной безопасности и теории управления Ульяновского государственного университета.

Россия, 432017, г. Ульяновск, ул. Лъва Толстого, 42, УлГУ.

\section{2. Токмаков Станислав Владимирович}

выпускник магистратуры факультета математики, информационных и авиационных технологий Ульяновского государственного университета.

Россия, 432017, г. Ульлновск, ул. Лъва Толстого, 42, УлГУ. 


\title{
ON USING NEUROCONTROL WITH DELAY IN OUTPUT FEEDBACK STABILIZATION PROBLEM
}

\author{
Sedova Nataliya Olegovna \\ Professor at Information Security and Control Theory department, \\ Ulyanovsk State University \\ Russia, 432017, Ulyanovsk, Leo Tolstoy str., 42, UlSU. \\ Tokmakov Stanislav Vladimirovich \\ Graduate of Mathematics, Information and Aviation Technologies faculty, \\ Ulyanovsk State University \\ Russia, 432017, Ulyanovsk, Leo Tolstoy str., 42, UlSU. \\ Received 30.10.2019, revised 29.01.2020.

\begin{abstract}
A method is proposed for stabilizing an inverted pendulum on a cart by the feedback that is independent of the speed values. The possibility of using only coordinate values is provided by feedback delay. The design uses a neural network, assuming some stabilizing state control as known. The latter is constructed in the form of switching control, allowing arbitrary deviations of the pendulum from the desired upper position.
\end{abstract}

Keywords: output stabilization, mechanical system, neurocontrol.

\section{Citation}

Sedova N.O., Tokmakov S.V., "On using neurocontrol with delay in output feedback stabilization problem", Nechetkie Sistemy i Myagkie Vychisleniya [Fuzzy Systems and Soft Computing], 15:1 (2020), 26-42 (in Russian). https://doi.org/10.26456/fssc66

\section{References}

[1] Andreev A.S., Peregudova O.A., "Two-link manipulator control synthesis without velocity measuring", Avtomatizatsiya protsessov upravleniya [Automation of management processes], 42:4 (2015), 81-89 (in Russian).

[2] Fridman E., Shaikhet L.E., "Delay-induced stability of vector second-order systems via simple Lyapunov functionals", Automatica, 74 (2016), 288-296.

[3] Roose A.I., Yahya S., Al-Rizzo H., "Fuzzy-logic control of an inverted pendulum on a cart", Computers and Electrical Engineering, 61 (2017), 31-47.

[4] Dotoli M., Lino P., Turchiano B., "A decoupled fuzzy sliding mode approach to swing-up and stabilize an inverted pendulum", IFAC Proceedings Volumes, 36:18 (2003), 113-118.

[5] Mladenov V., "Application of neural networks for control of inverted pendulum", WSEAS Transactions on circuits and systems, 10:2 (2011), 49-58.

[6] Zubov V.I., Lektsii po teorii upravleniya [Lectures on control theory], Nauka Publ., Moscow, 1975 (in Russian). 
[7] Tokmakov S.V., "On stabilization of linear systems by delayed feedback", Uchenye zapiski UlGU. Seriya: Matematika i informatsionnye tekhnologii [The Scientific Notes of the UlSU. Series: Mathematics and information technology], 2018, № 1, 98-108 (in Russian).

[8] Chenzhu Ch., Nonlinear control of an inverted pendulum, Graduate qualification paper (master's dissertation), Saint Petersburg Electrotechnical University "LETI", 2016 (in Russian). 